\title{
In-situ Lorentz TEM Observation of Magnetization Process in Fe-Pd Alloy
}

\author{
Noel T. Nuhfer, Lisha Wang and David E. Laughlin
}

Department of Materials Science and Engineering, Carnegie Mellon University, Pittsburgh, Pennsylvania 15213

Materials with high uniaxial anisotropy energy $\left(\mathrm{K}_{\mathrm{u}}\right)$, such as Fe-Pd and Fe-Pt alloys, are attractive for hard magnets and ultrahigh density magnetic recording applications [1]. However, the magnetic properties of these materials are very sensitive to the thermo-mechanical processing and the resultant microstructure. For example, although FePd alloy with stoichiometric composition has high anisotropy field $\left(\mathrm{H}_{\mathrm{K}}=33 \mathrm{kOe}\right)$, the highest coercivity reported for the bulk FePd is less than $1000 \mathrm{Oe}$ [2]. It has been shown that in these materials, a multi-domain structure is formed as a result of disorder order transformation and magnetic phase transformation. The magnetic domains (due to the ferromagnets demagnetization) are coupled with the structural domains (due to the disorder-order transformation) and hence influence the magnetic properties [3]. In order to understand the coercivity mechanism, it becomes necessary to observe the interaction between the magnetic domain structure and the microstructure during the magnetization process. In the present work, in-situ Lorentz TEM is used to study the magnetization process in Fe-Pd alloy.

The Fe55at. \%Pd alloy used in this study was prepared by the arc-melting process. The bulk Fe-Pd alloy was homogenized at $860^{\circ} \mathrm{C}$ for 24 hours and further annealed at $500^{\circ} \mathrm{C}$ for 3 hours. From the previous microstructural characterization, we know this specimen has mainly a polytwinned structure [4]. The polytwinned microstructure contains a hierarchy of twin variants that are formed to relieve the lattice mismatch strain. The coercivity of the material was measured to be about 155 Oe. Thin foils for TEM observation were electro-polished in a twinjet polisher using 25 Voltage at $0^{\circ} \mathrm{C}$. The solution consisted of $82 \mathrm{vol} . \%$ glacial acetic acid, 9 vol. \% perchloric acid and 9 vol. $\%$ ethanol [2].

The Lorentz TEM microscopy was carried out in Tecnai F20 microscope equipped with a Gatan image filter. In the Lorentz TEM mode, the objective lens is turned off and the specimen sits in a field free region. The advantage of the using Tacnai F20 microscope is that it is also equipped with a Lorentz lens and therefore can gradually increase the objective lens current. The magnetic field generated by the objective lens will then magnetize the TEM specimen inside the microscope. The maximum magnetic field generated by the objective lens is about $1.97 \mathrm{~T}$ [5]. By recording the strength of the objective lens, we can calculate the magnetic field applied on the specimen.

During the in-situ magnetization experiment, we increased the magnetic field at the rate of 15 Oe/step and observed the domain wall motion. When the domain wall moves, we record the strength of the objective lens and also imaged the magnetic domain structure by using Fresnel imaging mode. The results are shown in figure 1. In this figure, we can see that there are two types of magnetic domain walls: one is the magnetic micro-domain wall along the $\{110\}$ twin boundary; the other is the magnetic zig-zag macrodomain wall that intersects the twin boundaries. The magnetic macrodomain walls move as the magnetic field increases. The motion of the domain walls is collective, that is, the zig-zag domain walls remain continuous during the wall motion to avoid magnetostatic charges. This is consistent with the simulation results by Kazaryan et al. [6]. In the 
Lorentz microscopy Fresnel mode, the magnetic domain wall is imaged as alternate bright and dark lines. Comparing the region $\mathrm{A}$ in figure $1 \mathrm{a}, \mathrm{b}$ and $\mathrm{c}$ and region $\mathrm{B}$ in figure $1 \mathrm{~d}, \mathrm{e}, \mathrm{f}$, we find that the domain walls with opposite contrast are moving towards each other and finally disappear. At some stages of magnetization (Figure $1 \mathrm{~b}, \mathrm{c}, \mathrm{d}, \mathrm{e}$ ), the domain walls are pinned at the twin boundaries. The low coercivity of the specimen indicates that the pinning force of the twin boundaries is small. In fact, comparing figure 1d and 1e, we find that the domain wall (DW) pinned at a twin boundary moves at the magnetic field increment of $51 \mathrm{Oe}$. The apparent large magnification in figure $1 \mathrm{f}$ is due to the increased objective lens current. For the magnetic microdomain wall, we find that due to the high uniaxial anisotropy energy of the tetragonal phase, the microdomain wall does not move even at a magnetic field of 8900 Oe (figure 1f).

\section{Reference:}

[1] D. Weller, A. Moser, L. Folks, M. E. Best, W. Lee, M. F. Toney, M. Schwickert, J-U. Theiele, M. F. Doerner, IEEE Trans. Mag. 36 (2000), 10

[2] B. Zhang, W. Soffa, Phys. Stat. Sol. (a) 131 (1992), 707

[3] N. I. Vlasova, G. S. Kandaurova, N. N. Shchegoleva, Fiz. Met. Metalloved. 90 (2000), 31

[4] Lisha Wang, David E. Laughlin, Y. Wang, A. G. Khachaturyan, J. App. Phy. 93 (2003) (to be published)

[5] FEI company

[6] A. Kazaryan, Y. Wang, Yongmei M. Jin, Yu U. Wang, A. G. Khachaturyan, Lisha Wang, David E. Laughlin, J. App. Phy. 92 (2002), 7408

[7] This research was supported by the National Science Foundation under grant DMR-9905723.
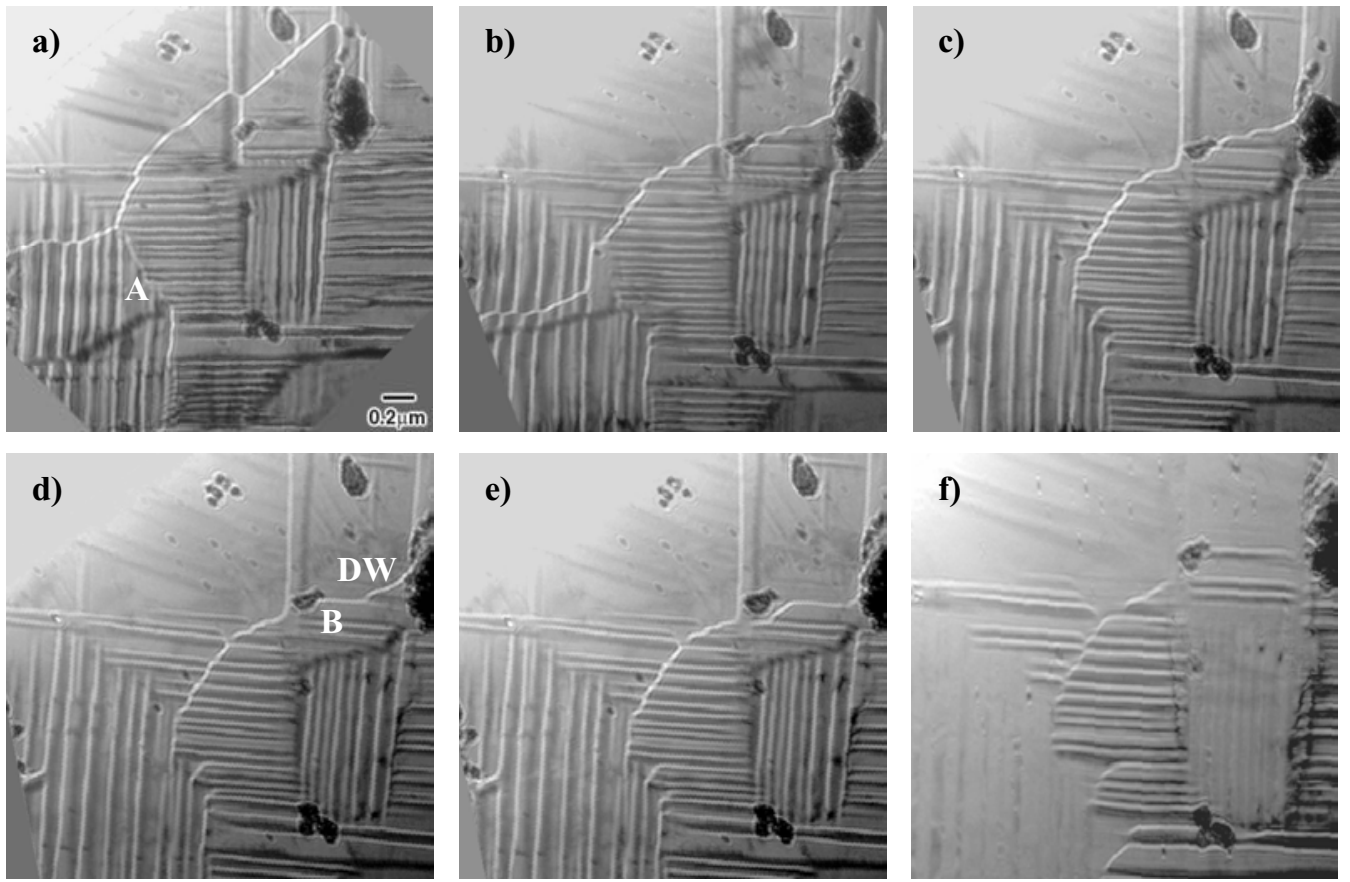

Figure 1. The In-situ Lorentz TEM observation of the magnetization process in Fe55at\%Pd alloy. The images are taken by using Fresnel imaging mode in the Lorentz microscopy. a) The magnetic field in the objective lens is approximately $0 \mathrm{Oe}, \mathrm{b}) 4500 \mathrm{Oe}$, c) $5115 \mathrm{Oe}$, d) $6019 \mathrm{Oe}$, e) $6070 \mathrm{Oe}$, f) $8900 \mathrm{Oe}$ 